\title{
Domain-Specific Backlinking Services in the Web of Data
}

\author{
Manuel Salvadores*, Gianluca Correndo*, Martin Szomszor ${ }^{\dagger}$, \\ *Yang Yang, Nick Gibbins*, Ian Millard*, Hugh Glaser*, and Nigel Shadbolt* \\ ${ }^{*}$ IAM Group, School of Electronics and Computer Science, University of Southampton, UK \\ $\dagger$ City eHealth Research Centre, City University London, UK \\ \{ms8,gc3,yy1402,nmg,icm,hg,nrs\}@ecs.soton.ac.uk,martin.szomszor@city.ac.uk
}

\begin{abstract}
This paper describes an Open Linked Data backlinking service, a generic architecture component to support the discovery of useful links between items across highly connected data sets. Using Public Sector Information (PSI) currently available as Linked Data, we demonstrate that contemporary publishing practices do not adequately support the ability to navigate or automatically traverse between resources published by different vendors, or the capacity to discovery information relevant to a particular URI. Although some useful services in this area have been developed, such as large triple indexes of published data, and the collection of sameAs relationships between individuals, we believe that an important component is missing: a mechanism to discover the backlinks to relevant resources that cannot be found by direct URI resolution. We present the implementation of such a component, integrating data from various PSI sources. We outline the possibility of exploiting semantics between graphs. We also evaluate our approach by measuring the potential information gained from a backlinking service, and the integration of backlinks with a co-reference service.
\end{abstract}

\section{INTRODUCTION}

The Open Linked Data (LOD) Initiative represents a collaborative effort to publish and link large amounts of data over the web using standard protocols and agreed representations. Much of this effort is centered on the premise that URIs are resolvable [3] and return RDF data that subsequently links it to other data items, creating a large Web of Data (WoD). In the formative years, Linked Data publishing was rolled out across a variety of domains, including entertainment, science, encyclopedia, government data, etc. It is anticipated that once the number of items exposed reaches a critical mass, reuse of data will become increasingly popular, fostering a new paradigm for the publishing and consumption of data, much like the Web 2.0 revolution did for REST [9], XML, and SOAP. In anticipation of this moment, our attention is drawn towards the additional architectural components that must be in place to support the consumers of Linked Data.

In the WoD vision, links between URIs from different publishers are particularly important since they are the ones that allow new data to be incorporated into the current discourse. For example, they are often used to express that different URIs on the Semantic Web are in fact referring to the same thing, usually with the owl:sameAs predicate.
Knowledge of this type of relationship increases the potential for reuse since information from previously unknown sources is now accessible. Some believe the onus is of the publisher to discover, create and publish links from their dataset to others in order to make their data more valuable. Other scenarios have been proposed [11] where specialised co-reference services, such as sameas.org, manage these links on behalf on the publisher. In any case, we can expect more and more of this linking data to be made available as the number of LOD publishers increases.

In a recent LOD exercise [15], we built an application to integrate data from various public sector information sources, such as the UK Government, the UK Ordnance Survey $^{1}$ and DBPedia ${ }^{2}$. The aim was to present an overview of a geographical location in terms of the crime, health, education, and traffic statistics associated with it. During this exercise, we found that following only LOD principles of i) resolve URI, ii) parse data, iii) follow links to other URIs, often meant we missed crucial joining data that would allow us to integrate information from different sources.

Although in some cases it is possible to discover additional locations in which a URI is used, for example by using the voiD Vocabulary [2] to discover SPARQL endpoints that can be queried, in the general case it is not possible using URI resolution alone because links are only uni-directional. If an RDF graph resolved from $U R I_{1}$ contains a reference to $U R I_{2}$, but the graph from $U R I_{2}$ has no reference to $U R I_{1}$, it is impossible to discover the relationship between the two URIs by resolution if only $U R I_{2}$ is known.

In this paper, we present our Backlinking Service, a generic component to support the publishing and discovery of useful links between URIs from different Linked Data publishers using only URI resolution. We begin in Section II with a review of the current state-of-the art. Section III focuses on the backlinking problem with respect to Linked Data and presents a motivating example for why a backlinking service is required. Section IV describes our implementation of a backlinking service and how it can be integrated with a co-reference service to maximize its utility. Our contribution is evaluated in Section V where the benefit

\footnotetext{
${ }^{1}$ http://www.ordnancesurvey.co.uk The mapping agency for Great Britain

${ }^{2} \mathrm{http} / / /$ dbpedia.org a Semantic Web representation of Wikipedia
} 
of our backlinking service is tested with UK PSI data. In Section VI our conclusions and future work are presented.

\section{BACKGROUND}

The World Wide Web (WWW) is a directed graph of documents (i.e. links that connect nodes in the hypergraph are unidirectional) and as such is asymmetric. This influences both its navigability and the possibility of information discovery. Historically some of the first hypertext systems implemented bidirectional links to freely enable navigation in both directions (e.g. Enquire, Microcosm [10] and Xanadu [14]). The possibility of having backlinks was also taken into account in the design of link topology ${ }^{3}$ in the infancy of the WWW and since then proposals have been made for building such links off line [6], [17].

There are already a few proposals born within the hypertext community for gathering backlinks (e.g. [5], [6] or the Google search engine using the "link" option), but these proposals are not aimed at the Semantic Web, and target the traditional Web of documents. Often these proposals rely on a centralised service which scrapes the online documents, analyses the embedded links, indexes the results, and offers them as a service. Such centralized approaches can suffer to some extent from scalability issues [13] that will affect the performance of the offered services as the size of the analysed document grows.

Distributed approaches proposed in the past [18] relied on logging the Referer HTTP header (see [8], section 14.36) during the usual document serving activity by a web server. Such information can then be stored in distributed databases and used afterwards. The pertinent question here is then who should be in charge of storing and managing such information? The WWW topology has shown to follow power-laws [1] and if the Semantic Web information resources topology would do the same (and it does at least for what concerns the ontology usage [4]) then few sites/resources will have to manage a huge amount of back links information without much control over future links published by the community.

The Linked Data community is pursuing the use of the Web for publishing and connecting data adopting RDF as a common data model, URIs for identifying resources over the Web and HTTP as a mechanism for retrieving information about those resources. The main topology of the Semantic Web information network is therefore very similar to that of the WWW, containing links that connect pieces of information that are discoverable and browsable in only one direction. The use of data sets such as geonames and dbpedia that are emerging as common data hubs for providing context to newly Linked Data resources will pose in the future problems of data retrieval if those data sets will be adopted by users as their entry point for data searches.

\footnotetext{
${ }^{3}$ http://www.w3.org/DesignIssues/BuildingBackLinks.html last access $24 / 03 / 2010$
}

New technologies have been developed by the Semantic Web community to provide more diverse access to Linked Data sets. SPARQL endpoints are sometimes provided for running queries against data sets, and indexing services for linked open data (e.g. sindice.com) can support the discovery of backlinks. Sindice [16] is a lookup index over RDF documents crawled on the Semantic Web, and it allows users and software agents to discover documents containing information about a given resource. Such centralized services can be used in order to discover links pointing to a resource by just collecting all pertinent documents, parsing them and recording the triples that explicitly mention the resource of interest. The performance of discovering backlinks in this fashion greatly depends on the size of the documents returned by the lookup process. The greater the number of triples contained in a single document, the heavier the process of parsing and querying it is and this must be taken into account in order to design a scalable service.

Significant help to discover backlinks could be provided by the extensive use of voiD ${ }^{4}[2]$ descriptors. VoiD descriptors give a precise notion of the topology of the linked data cloud and could be used in order to narrow the rose of possible data sets that could provide incoming links to a resource of interest. However such knowledge should be coupled by the capability, provided by those data sets, to return the incoming links. URI resolution is out of the question because the URIs to resolve cannot be known in advance; they are the very object of this research. Therefore a SPARQL endpoint or a lighter service that can return such links must be implemented by each data set, and "voiD + SPARQL" can be considered a backlinking service. However, we cannot expect all the data sets in the WoD to provide a SPARQL end-point (expensive to maintain) and use voiD. (from the data consumer perspective, not trivial to use).

Semantic Web approaches for discovering backlinks in the Web of Data are still missing. Although there are some tools that can provide backlinks (such as sindice.com), these operate at the document level and they do not facilitate the discovery of backlinks at the graph level. This means that current solutions are not adequate for the WoD because their results give back documents but not resolvable URIs.

In contrast to the methods outlined above, in our work we have focussed on the basic tenets of Linked Data in trying to provide a lightweight solution to the problem of retrieving backlinks from a resource of interest, relying on resolvable URIs only. Another import characteristic of our solution is that our backlinking service is itself Linked Data, therefore it is seamlessly integrated and can be seen as a Linked Data layer on top of the current WoD.

Notably, the solution proposed cannot claim to be distributed, although this is an intended outcome of the work.

\footnotetext{
${ }^{4}$ http://semanticweb.org/wiki/VoiD last accessed 24/03/2010
} 


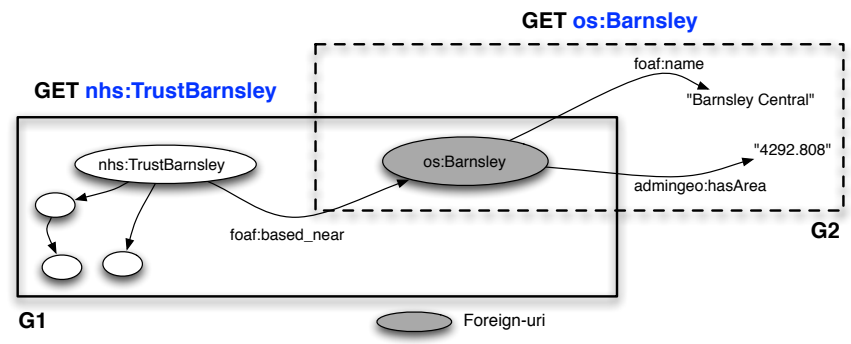

Figure 1. foreign URI (with respect to nhs.psi.enakting.org dataset)

While the amount of data analysed and indexed by this service so far is relatively modest, we can already demonstrate the benefits of domain-specific backlink services in the WoD.

\section{MOTIVATION}

The four Linked Data principles [3] promote RDF as the standard data model and encourages the community to create a decentralized RDF graph at a Web scale, the WoD. We have identified a navigability issue in the WoD and this paper proposes a solution to improve the connectivity of the RDF graph that represents the WoD. The solution we propose is a lightweight service designed to facilitate backward navigation between different data sets. Backward navigations are broken if the object of the triple to be followed comes from a different data set. Figure 1 shows two RDF graphs that can be obtained by resolution of two URIs:

- $G_{1}$ by HTTP resolution of nsh:TrustBarnsley ${ }^{5}$

- $G_{2}$ by HTTP resolution of os: Barnsley 6

nsh:TrustBarnsley represents a hospital in the UK National Health Service (NHS), and os:Barnsley is a resource that represents a UK geographic location. os: Barnsley is a foreign URI in $G_{1}$ because its domain is not the same as the publisher (nhs.psi.enakting.org). At this point we can say that "os:Barnsley is a foreign URI in the graph defined by nsh:TrustBarnsley". In general, we can define the term foreign URI as:

"A URI $X$ is considered foreign in a RDF Graph $G$ if exists a triple $(s, p, X)$ in $G$ and domain $(x)<>\operatorname{domain}(G)$ "

Foreign URIs represent a barrier when navigating the WoD and it is a pattern that happens quite often. This is due to the fact that there are many statements with foreign URIs generated by join point data sets. For instance a geographic data set produces many of this type of statements. This case is a clear example of broken navigation that happens now in the WoD.

\footnotetext{
${ }^{5}$ http://nhs.psi.enakting.org/id/A_RFF

${ }^{6} \mathrm{http} / / /$ data.ordnancesurvey.co.uk/id/7000000000024753
}

\section{A. Broken Navigation with foreign URIs}

Foreign URIs make data discovery difficult because it is not possible to navigate the RDF documents of the WoD bidirectionally. Let us assume we want to discover alternative resources or additional facts concerning the URI dbpedia:Barnsley ${ }^{7}$. Which happens to be the equivalent os:Barnsley. This type of equivalence can be looked up in the WoD through co-reference systems. A co-reference system, such as sameAs.org, is a service that enables the discovery of sets of resources that are equivalent. These services increase considerably the connectivity of the WoD facilitating the integration of data sets. sameAs.org is a domain-independent service for the WoD. Our work pursues better connectivity in the WoD and we consider that coreference systems are partially achieving this outcome, and they resolve it partially because co-reference systems do not resolve backward navigation with Foreign URIs.

The broken navigation between nsh:TrustBarnsley and dbpedia:Barnsley gets reflected through the following example. To summarize, the following triples are decentralised in separate graphs of the WoD:

- $T_{1}$ (nsh:TrustBarnsley, foaf:based_near, os:Barnsley) Obtained by direct resolution of nsh:TrustBarnsley.

- $T_{2}$ (os:Barnsley, owl:sameAs, dbpedia:Barnsley) Obtained via RESTFul query to sameAs.org.

- $T_{3}$ (dbpedia:Barnsley, foaf:name, "Barnsley Central") Obtained by direct resolution of dbpedia:Barnsley.

For clarity, let's assume the following symbol equivalences: $X=n s h:$ TrustBarnsley, $Y=o s:$ Barnsley and $\mathrm{Z}=$ dbpedia:Barnsley. So if we take $T_{1}, T_{2}$ and $T_{3}$ as a story board to navigate through them, then we realize that we can perform the navigation forward $(X \rightarrow Y \rightarrow Z)$ but not backwards $(Z \rightarrow Y \nrightarrow X)$. When navigating backwards, starting from $Z, X$ is not reachable by recursive HTTP resolution. This happens because the publisher of $Y$ (os:Barnsley) is not meant to return URIs from other data sets that link to its resources. Current practice only recommends to provide backlinks within the same dataset. Therefore we will not find any triples mentioning $X$ in the RDF graph represented by $Y$, we denote this case by $Y \nrightarrow X$. In this case, the bidirectional navigation can be represented as:

$$
(X \rightarrow Y \rightarrow Z)+(Z \rightarrow Y \nrightarrow X)=(X \rightarrow Y \leftrightarrow Z)
$$

Even though this backward navigation is broken it is up to the publishers to avoid this issue by keeping all the triples they produce with URIs that belong to their domain. For those cases co-reference systems are an optimal solution to traverse between resources from different data sets.

\footnotetext{
${ }^{7}$ http://dbpedia.org/page/Barnsley_Central
} 


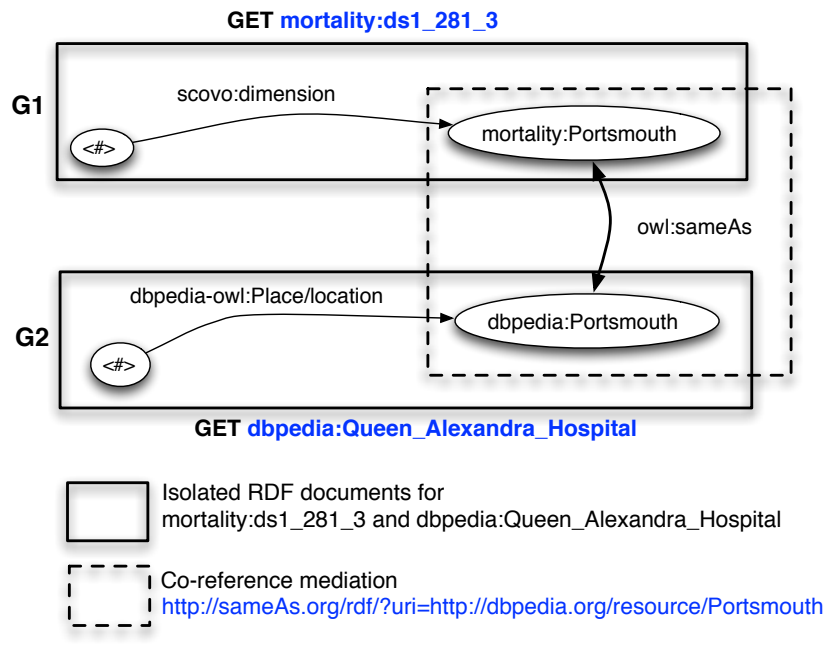

Figure 2. Co-reference Systems Approach

\section{B. Co-reference System Approach}

The practice for publishing Linked Data recommends that backlinks are published for the URIs that belong to the same data set $^{8}$. Let us assume two URIs (mortality:ds1_281_39, dbpedia:Queen_AH ${ }^{10}$ ) produce RDF graphs without foreign URIs. For these we will demonstrate that a co-reference service such sameAs.org resolves the navigation problem.

mortality:ds1_281_3 represents mortality statistics in SCOVO[12] for the UK region of Portsmouth, dbpedia:Queen_AH is the resource that represents a hospital located in the same geographical region. The RDF graphs retrieved by resolving these URIs are not linked together by themselves because each of the publishers, following the Concise Bounded Description (CBD) ${ }^{11}$, only returns a subset of the RDF graph within their data sets.

This problem can be solved by using a co-reference system. Both graphs are pointing to URIs that are located in a common bundle in sameAs.org therefore the two RDF graphs are now part of a connected RDF graph and it is possible to navigate automatically between them.

Figure 2 shows how the co-reference system connects bidirectionally the isolated RDF graphs. In this case, it is possible to navigate from mortality:ds1_281_3 to dbpedia: Queen_AH and vice-versa by just making recursive HTTP resolutions. This example works based on two assumptions:

- The publishers from both domains describe their resources providing backlinks to the subjects from the

${ }^{8} \mathrm{http} / / /$ www4.wiwiss.fu-berlin.de/bizer/pub/LinkedDataTutorial/ last accessed 24/03/2010

${ }^{9}$ http://mortality.psi.enakting.org/id/ds1_281_3

${ }^{10} \mathrm{http}: / / \mathrm{dbpedia.org} /$ resource/Queen_Alexandra_Hospital

${ }^{11}$ http://www.w3.org/Submission/CBD/ last accessed 24/03/2010 same data set following the CBD mechanism.

- The publishers avoid making statements that include foreign URIs.

Taking into account these two assumptions the following example represents a possible story board of HTTP resolutions. Let's assume the following symbol equivalence: $\mathrm{X}$ = dbpedia:Queen_AH, Y = dbpedia:Portsmouth, $\mathrm{Z}=$ mortality: Portsmouth, $\mathrm{T}=$ mortality:ds1_281_3.

Looking at the triples represented in Figure 2, we can see that none of the URIs $X, Y, Z$ and $T$ represented in the graphs $G_{1}$ and $G_{2}$ are foreign URIs because they all come from the same domain as the graph they belong to. Therefore, following the practice, the resolution of $X, Y, Z$ and $T$ will provide the backlinks within the same data set and using a co-reference service as mediator we can perform bidirectional navigation between any pair of URIs from $G_{1}$ and $G_{2}$. We can denote this via:

$$
(X \leftrightarrow Y)+(Z \leftrightarrow T)+(Y \equiv Z)=(X \leftrightarrow Y \leftrightarrow T)
$$

(or)

$$
(X \leftrightarrow Y)+(Z \leftrightarrow T)+(Y \equiv Z)=(X \leftrightarrow Z \leftrightarrow T)
$$

Since $X$ and $Y$ are URIs that belong to the same data set we can assume the publisher will return backlinks between them when resolving any of them. The same assumption applies between $Z$ and $T$. The mediation piece $(Y \equiv Z)$ represents the co-reference discovery provided by sameAs.org.

This navigation example relies on the assumption that RDF documents in the WoD do not contain foreign URIs. This assumption does not hold in reality because the Linked Data community evangelizes that to realise the power of the WoD one should wherever possible re-use URIs rather than duplicating descriptions of entities or concepts. Therefore we predict that WoD will experience a rapid increase of foreign URI linkages as the number of data hubs increase.

The main motivation of this research is to enable the discovery of foreign URI linkages by designing and implementing a lightweight backlinking service. In order to probe the benefit of such a service we have focussed on a specific domain, the UK Public Sector Information domain.

\section{Public Sector Information Backlinking SERVICE}

To facilitate consumers of the WoD in the discovery of foreign-URIs, we have designed a backlinking service that keeps track of this special type of link pattern. We have implemented this service for a number of UK PSI data sets currently available as Linked Data. The service, located at http://backlinks.psi.enakting. org $^{12}$, is designed according to three basic principles:

\footnotetext{
${ }^{12}$ Refer to http://backlinks.psi.enakting.org for usage examples and API documentation
} 


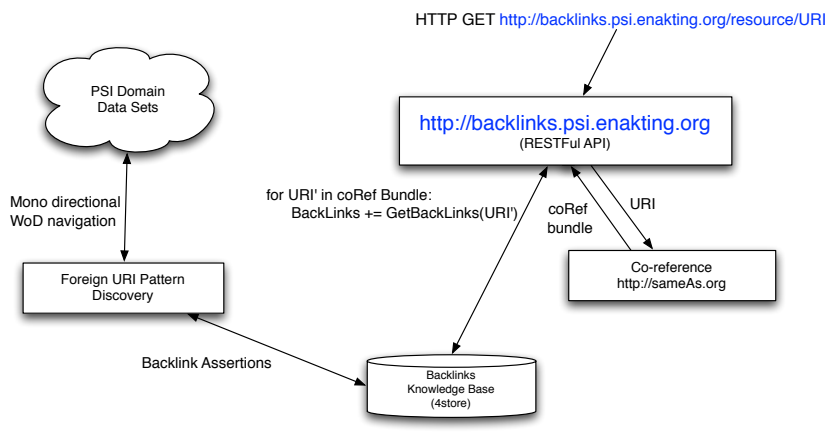

Figure 3. High-level Architecture

- Lightweight Service: The service must be easy to use and resolve a specific problem. A backlinking service is a component of the WoD specifically design to resolve a discovery problem, it is not a search engine and it is not a large WoD index.

- Linked Data Compatible: The backlinking service should be compatible with current Linking Open Data best practises, with all requests supporting URI HTTP resolution and content negotiation. Software processes should be able to easily interact with the service, and to retrieve results in a number of useful formats including RDF. As a result this service shall form another layer on top of the $\mathrm{WoD}$, while existing Linked Data applications can embrace the knowledge contained at extremely low cost.

- Co-reference Support: to extend the WoD graph coverage the backlinking service is integrated with a coreference system. This approach allows us to reach data sets from across unbounded WoD.

Figure 3 is a visual overview of the architecture, and is composed of three parts:

- Foreign URI Pattern Discovery Component: This is the component responsible for automatically navigating the PSI data sets from the $\mathrm{WoD}$ and identifying foreign URIs. This component crawls the WoD retrieving all the foreign URIs found in the data sets under the study. It resolves all the RDF documents from a starting (or input) list of URIs and inspects each document returned to identify triples in which the object is a foreign URI. For every foreign URI found we assert a rdfs:seeAlso statement into the knowledge base. The seeAlso statement is a triple that points to the original URI in case of backward navigation. For instance, if the service was analysing nsh:TrustBarnsley (see Section III) then we would discover that the document returned by resolving that URI contains a triple in which os:Barnsley is in the object position, i.e. os:Barnsley is a foreign URI in this context. If a client were seeking information about os:Barnsley then it may wish to discover the information about that concept contained within the document retrieved when resolving nsh:TrustBarnsley. As a result, the corresponding assertion into the backlinks knowledge base is:

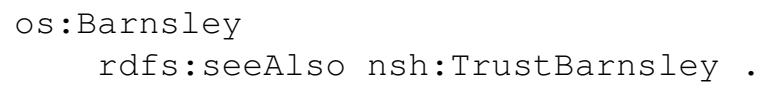

Which follows the pattern:

$<$ FOREIGN-URI > rdfs: seeAlso <LOCAL-URI>

- RESTFul API as Front-end Service: Access to the service is provided by a RESTFul API that accepts requests by simple HTTP GETs. The interface is: http://backlinks.psi.enakting.org/resource/URI Where URI is the resource for which we want to discover the backlinks. The service queries the knowledge base where the seeAlso statements were asserted and returns a document with all the backlinks. The output of the service can be obtained in JSON, RDF+XML, TURTLE or HTML, either through the Service URL or by specifying the accept header of the HTTP request ${ }^{13}$. The logic of this service is integrated with a coreference system extending its functionality to all the URIs in a sameAs bundle. Let us assume a sameAs bundle is made of three URIs:

$$
\text { sameAsBundle }=\left\{U R I_{1}, U R I_{2}, U R I_{3}\right\}
$$

and there is a set of two backlink assertions in our system:

$$
\begin{aligned}
& \left\{<U R I_{2}>\text { seeAlso }<U R I_{x}>\right\} \\
& \left\{<U R I_{2}>\text { seeAlso }<U R I_{y}>\right\}
\end{aligned}
$$

The system will return $\left\{U R I_{x}, U R I_{y}\right\}$ as backlinks when receiving any of the URIs part of the sameAsBundle as input URI.

- Knowledge Base: We have chosen to use 4store for the internal storage of the backlinks knowledge. While a number of mechanisms could have been employed, using an RDF based store offers significant benefits in terms of flexibility, and we already have an enterprise scale platform available for hosting the service.

The system records backlink metadata, such as the RDFS label and the RDF type(s) of every URI subject of a backlink. Hence, the complete output for a backlink in the system is as follows:

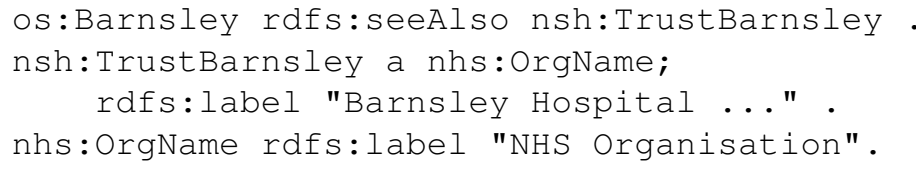

\footnotetext{
${ }^{13}$ More detailed documentation about the API can be found at http:// backlinks.psi.enakting.org
} 


\section{A. Geographic ad-hoc Semantics: an optional capability}

Many of the PSI data sets published so far are related to a spatial and temporal dimension, in other words, all data can be linked together by its spatial and temporal indexes. This is unsurprising, the spatial and temporal reasoning have always been considered to be an important part of commonsense reasoning in Artificial Intelligence. Pursuing a better connected WoD we developed a Geographical Service for the WoD. This service computes the closure of partonomies for the UK geography taking as source the Ordnance Survey Linked dataset. To improve the backlinking coverage we aim to get all the possible containments from all the dictionaries supported in the geographical service for a given URI. There is a natural outcome from this integration and it can be shown with the following example: when asking for backlinks connected to the URI dbpedia:Hampshire. Prior to the use of the geographical extension a request to retrieve backlinks for dbpedia:Hampshire would just give back 14 URIs. This same request when the geographical service is integrated returns 12345 resources $^{14}$ contained within Hampshire.

We have kept the decentralised nature of the Backlinking and Geographical services and the Backlinking Service performs HTTP requests to get the geographic containments. When the geography extension is enabled the Backlinking service gets the list of contained entities for the input URI and returns the backlinks connected to any URI part of those containments. In [7] is described such integration and the implementation of the Geographical Service.

This ad-hoc reasoning exploits the semantics of such contextual dimensions for easing entity retrieval and browsing. And, as the community identifies more semantics, we envision more of this type of reasoning services being integrated with the Backlinking service to improve the WoD navigability.

\section{EVALUATION}

Following Tim Berners-Lee's call for raw data now ${ }^{15}$ the UK Prime Minister decided to favour the opening of Public Sector information. The Public Sector data is retained, up to now, by central government, local councils, the NHS, police and education authorities and other governmental institutions. By releasing and giving open access to this information the government intends to increase its transparency and create economic and social capital. Our evaluation framework is based on data sets that are part of the government initiative, the Ordnance Survey and the EnAKTing project. The backlinking assertions have been sourced from the following data sets:

\footnotetext{
${ }^{14}$ Refer to http://backlinks.psi.enakting.org/help for a more graphical explanation.

${ }^{15} \mathrm{http}: / /$ www.ted.com/talks/tim_berners_lee_on_the_next_web.html last accessed 20/12/2009
}

- co2emission.psi.enakting.org: Statistical data about $\mathrm{CO} 2$ in the UK, the emissions are hierarchically typed by cause of emission and linked to the region where it was measure.

- energy.psi.enakting.org: This dataset represents the energy consumption for the road network in the UK between 2002 and 2007.

- population.psi.enakting.org: Statistical data about population in the UK segmentated by, age, gender and location between 2001 and 2007.

- nhs.psi.enakting.org: Statistical data about the number of patients waiting for a first outpatient appointment following a GP referral. NHS names that can be connected to its location, year of validity and weeks waited.

- mortality.psi.enakting.org: Statistical data about mortality. This data set is geographically segmented by UK regions and gender.

- crime.psi.enakting.org: Crime statistics provided by the UK Home Office ${ }^{16}$. These statistics are divided by region and type of crime.

- parliament.psi.enakting.org: Information about the UK Members of Parliament, their expenses, affiliations and votes. The original sources of this data are theyworkforyou.com and publicwhip.org.uk.

- data.ordnancesurvey.co.uk: The Ordnance Survey is the authoritative publisher of geographic information in the UK. This data set contains several interconnected hierarchies of types of regions in the UK such as Constituencies, Wards and Boroughs.

- *data.gov.uk: Under data.gov.uk the UK is publishing different data sets using Semantic Web technologies. We identified two data sets in data.gov . uk that are ready to be part of this study.

From all the selected data sets we have identified data.ordnancesurvey.co.uk as the the hub of linkages. This means that all the other data sets in some manner (either directly or through sameAs.org) link to the UK Geographic data set. All the data sets under the domain *.psi.enakting . org use the Ordnance Survey ontology, via the owl: sameAs alignments. Other datasets from $*$.data.gov.uk adopt the same ontology as their authoritative source for geographical information.

The presence of authoritative datasets, such as data.ordnancesurvey.co.uk, is a strong point for data integration and improves the connectivity of the WoD, but it also weakens the navigation since it generates a high level of foreign URI statements in RDF documents that reside in other domains. Our backlinking service allows the user or agent to navigate backwards from the authoritative data sets towards these RDF documents with foreign URIs.

\footnotetext{
${ }^{16}$ http://www.homeoffice.gov.uk/ last accessed 20/12/2009
} 


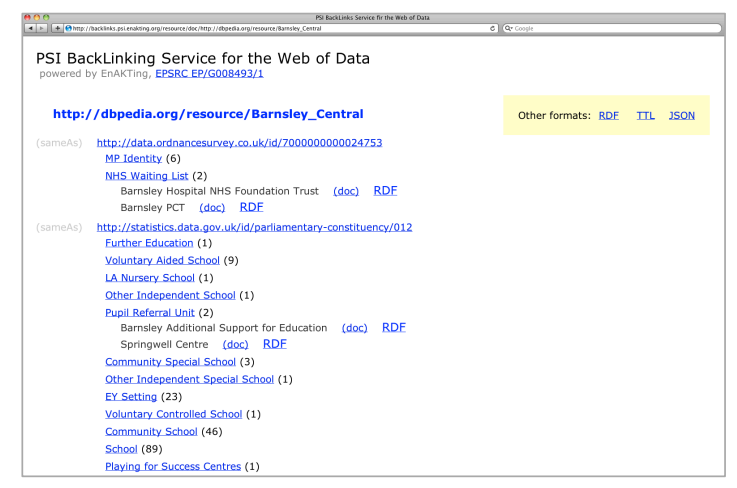

Figure 4. Backlinking Service for dbpedia:Barnsley from Section III-A

Following up from the broken navigation identified in Section III-A, Figure 4 shows the Backlinking Service output for dbpedia:Barnsley ${ }^{17}$. The Figure shows the HTML version of the backlinks returned by the system and their categorization by RDF type. The output of the service shows that for dbpedia:Barnsley we do not just reach information about hospitals but also education and parliament.

The provided backlinking service enhances the navigation of the WoD by augmenting the connectivity of the information network. It provides links to resources that were not reachable before. One metric that provides a concrete measurement of the added value of the service is the number of new links that the backlinking asserts into the WoD.

The added connectivity by our Backlinking service, for the case study of the UK public sector information, is depicted in Table I. The first column of the table represents the data sets from where a backlink exists. The data sets in the header represent the origin of the backlinks. The numbers are the occurrences of backlinks for each pair of data sets. The first two rows represent the original backlinks and the rest represent co-reference expansions reached trough sameAs.org. As an example we can say that from sws.geonames.org it is possible to navigate to 9345 resources from the education data set. It can be seen that the combination of a Backlinking service with a co-reference system is a powerful mechanism to improve the navigation from data hubs towards other data sets.

We complete the evaluation of the backlinking by developing a Web application ${ }^{18}$ that using the OpenSpace ${ }^{19}$ map API for the UK displays the data in a meaningful way for an end-user, see Figure 5. The application is accesible at http://map.psi.enakting.org and is just one of the possible usages of our Backlinking Service. We can

\footnotetext{
${ }^{17} \mathrm{http} / / /$ backlinks.psi.enakting.org/resource/doc/http://dbpedia.org/ resource/Barnsley_Central

${ }^{18}$ Refer to http://map.psi.enakting.org/how accessed 19/03/2010

${ }^{19} \mathrm{http}: / /$ openspace.ordnancesurvey.co.uk/openspace/ $19 / 03 / 2010$
}

consider this application "Linked Data Unbounded" because as more resources are discovered by the Backlinking service it will be able to integrate them seamlessly without any implementation or configuration effort.

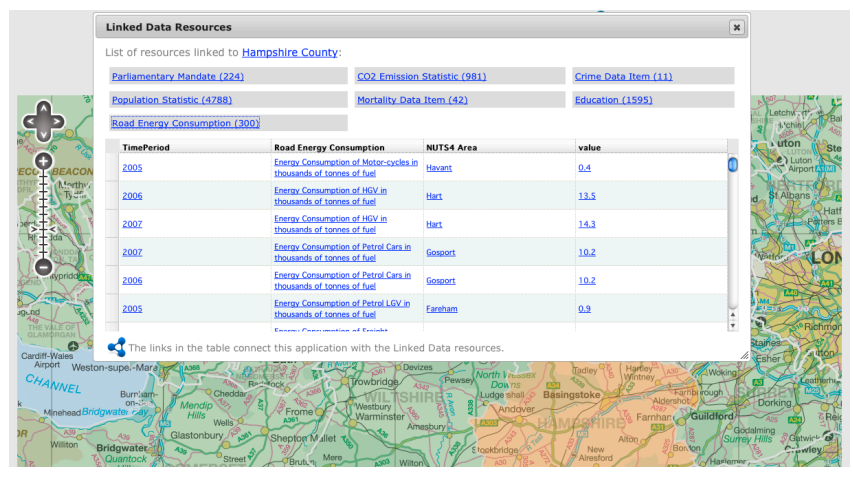

Figure 5. Map application consuming Backlinks for the region of Hampshire

\section{CONCLUSIONS}

In this paper, we have argued that the absence of backlinks from Foreign URIs in Open Linked Data results in a navigational gap. Using Public Sector Information (PSI), we provide motivating examples as to why backlinks from Foreign URIs are especially important for items that link data from multiple publishers. These hubs provide the bridge between information resources, supplying the essential links in the WoD that will maintain a Small World network property and allow consumers to combine previously disconnected pieces of data. To alleviate this navigation problem, we present our design of a generic light-weight backlinking service and describe our implementation over UK PSI. By making use of the sameas.org Co-reference Service, we are able not only to provide a service that returns Foreign URIs that link to a particular URI, but also those URIs that are equivalent. Finally we have also shown how the exploitation of adhoc semantics such as geographic containments can increase the data navigability in cases where a spatial dimension is attached to the information.

We have evaluated our work, demonstrating that a significant number of backlinking statements (e.g. over $1.4 \mathrm{M}$ statements linking statistics.data.gov.uk to education.psi. enakting.org) can be automatically generated and served to Linked Data consumers via a simple REST API. As an example of such backlinking exploitation we have developed a Web application that through a map interface is able to navigate the Web of Data displaying information from decentralized data sets.

\section{ACKNOWLEDGEMENTS}

This work was supported by the EnAKTing project funded by the Engineering and Physical Sciences Research Council under contract EP/G008493/1. 
Table I

DATASETS LINKAGE IMPROVEMENT STATISTICS

\begin{tabular}{|c|c|c|c|c|c|c|c|c|}
\hline & \multicolumn{8}{|c|}{ Backlink to } \\
\hline Backlink from & crime & mortality & education & parliament & nhs & energy & $\operatorname{co2}$ & population \\
\hline *statistics.data.gov.uk & 1280 & 4389 & 1444196 & 22830 & 240 & 38850 & 117487 & 418152 \\
\hline *data.ordnancesurvey.co.uk & 888 & 2376 & 226936 & 24398 & 256 & 20780 & 62422 & 421344 \\
\hline linkedgeodata.org & 19 & 54 & 7036 & 0 & 0 & 540 & 1580 & 0 \\
\hline sw.cyc.com & 11 & 9 & 475 & 0 & 0 & 60 & 172 & 0 \\
\hline unlocode.rkbexplorer.com & 11 & 15 & 950 & 0 & 0 & 120 & 344 & 0 \\
\hline rdf.freebase.com & 337 & 279 & 83094 & 11337 & 120 & 2190 & 6617 & 207480 \\
\hline airports.dataincubator.org & 1 & 3 & 505 & 0 & 0 & 30 & 90 & 0 \\
\hline dbpedia.org & 511 & 426 & 97945 & 11721 & 122 & 3480 & 10480 & 213864 \\
\hline www.twine.com & 0 & 3 & 252 & 0 & 0 & 20780 & 87 & 0 \\
\hline www.okkam.org & 0 & 6 & 475 & 0 & 0 & 30 & 172 & 0 \\
\hline guardian.dataincubator.org & 0 & 0 & 63520 & 11768 & 120 & 0 & 0 & 208677 \\
\hline revyu.com & 0 & 3 & 252 & 0 & 0 & 60 & 0 & 0 \\
\hline umbel.org & 205 & 183 & 16841 & 360 & 0 & 1650 & 4840 & 5187 \\
\hline os.rkbexplorer.com & 367 & 324 & 21613 & 0 & 0 & 2610 & 8035 & 0 \\
\hline sws.geonames.org & 32 & 93 & 9345 & 0 & 0 & 930 & 2759 & 0 \\
\hline sw.opencyc.org & 1635 & 1170 & 75720 & 30 & 0 & 8400 & 25480 & 1995 \\
\hline mpii.de & 250 & 255 & 19775 & 102 & 0 & 2250 & 6887 & 798 \\
\hline
\end{tabular}

\section{REFERENCES}

[1] Lada A. Adamic, Bernardo A. Huberman; A. Barabási, R. Albert, H. Jeong, and G. Bianconi;. Power-law distribution of the world wide web. Science, 287(5461):2115a+, March 2000 .

[2] K. Alexander, R. Cyganiak, M. Hausenblas, and J. Zhao. Describing Linked Datasets - On the Design and Usage of voiD, the 'Vocabulary of Interlinked Datasets'. In WWW 2009 Workshop: Linked Data on the Web (LDOW2009), Madrid, Spain, 2009.

[3] Tim Berners-Lee. Design issues: Linked data. http://www.w3.org/DesignIssues/LinkedData.html, 2006.

[4] Tim Berners-Lee and Lalana Kagal. The fractal nature of the semantic web. AI Magazine, 29(3):29-34, 2008.

[5] Krishna Bharat, Andrei Broder, Monika Henzinger, and Puneet Kumar. The connectivity server: fast access to linkage information on the web. In proceedings of the $7^{\text {th }}$ International World Wide Web Conference, 1997.

[6] Soumen Chakrabarti, David A. Gibson, and Kevin S. Mccurley. Surfing the web backwards. In In: Proc. of $W W W 8$ Conference, pages 1679-1693, 1999.

[7] Gianluca Correndo, Manuel Salvadores, Yang Yang, Nick Gibbins, and Nigel Shadbolt. Geographical service: a compass for the web of data. In WWW2010 Workshop: Linked Data on the Web (LDOW2010), April 2010.

[8] R. Fielding, J. Gettys, J. Mogul, H. Frystyk, L. Masinter, P. Leach, and T. Berners-Lee. Hypertext transfer protocol http/1.1. RFC 2616 (Draft Standard), June 1999. Updated by RFC 2817.

[9] Roy T. Fielding. Architectural Styles and the Design of Network-based Software Architectures. PhD thesis, University of California, Irvine, 2000.
[10] Andrew M. Fountain, Wendy Hall, Ian Heath, and Hugh Davis. Microcosm: An open model for hypermedia with dynamic linking. In A Rizk, N Streitz, and J Andre, editors, Hypertext: Concepts, Systems and Applications, Proceedings of ECHT'90, Paris, November 1990, pages 298-311. Cambridge University Press, 1990.

[11] Hugh Glaser, Afraz Jaffri, and Ian Millard. Managing coreference on the semantic web. In WWW2009 Workshop: Linked Data on the Web (LDOW2009), April 2009.

[12] Michael Hausenblas, Wolfgang Halb, Yves Raimond, Lee Feigenbaum, and Danny Ayers. SCOVO: Using statistics on the web of data. In ESWC, volume 5554 of Lecture Notes in Computer Science, pages 708-722. Springer, 2009.

[13] Frank Kappe. A scalable architecture for maintaining referential integrity in distributed information systems. The Journal of Universal Computer Science - JUCS, 1(2):84-104, 1995.

[14] Theodor H. Nelson. Literary machines. T. Nelson, 1981.

[15] Tope Omitola, Christos Koumenides, Igor Popov, Yang Yang, Manuel Salvadores, Gianluca Correndo, Tim Berners-Lee, Nick Gibbins, Wendy Hall, mc schraefel, and Nigel Shadbolt. Put in your postcode, out comes the data: A case study. In 7th Extended Semantic Web Conference, April May 2010.

[16] Eyal Oren, Renaud Delbru, Michele Catasta, Richard Cyganiak, Holger Stenzhorn, and Giovanni Tummarello. Sindice.com: a document-oriented lookup index for open linked data. IJMSO, 3(1):37-52, 2008.

[17] James E. Pitkow and R. Kipp Jones. Supporting the web: A distributed hyperlink database system. Computer Networks and ISDN Systems, 28(7-11):981 - 991, 1996. Proceedings of the Fifth International World Wide Web Conference 6-10 May 1996.

[18] James E. Pitkow and R. Kipp Jones. Supporting the web: a distributed hyperlink database system. Comput. Netw. ISDN Syst., 28(7-11):981-991, 1996. 